\section{QUALITY OF LIFE FOLLOWING A ROAD TRAFFIC INJURY: A SYSTEMATIC LITERATURE REVIEW}

${ }^{1}$ Ritva Rissanen, ${ }^{1,2}$ Hans-Yngve Berg, ${ }^{1}$ Marie Hasselberg. 'Karolinska Institutet, Sweden; ${ }^{2}$ Swedish Transport Agency, Sweden

\subsection{6/injuryprev-2016-042156.593}

Background Every year, 1.3 million people are killed and up to 50 million are injured in road traffic accidents worldwide. The burden of road traffic injury has shifted from the premature death to a reduced quality of life (QoL) for those injured, therefore it is important to understand the effect of road traffic injury (RTI) on QoL. We aimed to assess and conclude the current knowledge about the relationship between RTI and QoL.

Methods A systematic review of the literature on QoL after an RTI, in both adults and paediatric populations, from 3 databases (Pubmed, PsychInfo and SafetyLit) over the last fourteen years was undertaken. The methodological quality of the studies was assessed according to the Newcastle-Ottawa Quality Assessment Scale.

Results Nineteen articles were included and assessed for quality. In general, the QoL scores of those injured were similar to population norms at the first assessment, followed by a drop from the first assessment to the second assessment. The majority of the participants reported an increase of QoL from the second assessment to the third assessment but they never reached the population norms at the last follow-up (range 6 weeks to 2 years). Age, gender, socioeconomic status, injury severity, injury type, and PTSD were associated with poorer QoL.

Conclusions The available literature regarding the QoL of persons injured in road traffic accidents is heterogeneous in regards to aims and tools used for assessment. Our review confirmed that independent of how QoL was measured, the overall QoL is significantly reduced after an RTI compared to the general population norms. Persons who are older, of female gender, lower socioeconomic status, diagnosed with PTSD, with more severe injuries or injuries to the lower limbs are more vulnerable to loss of QoL following an RTI compared to other patient groups injured in road traffic accidents.

\section{DEVELOPMENT AND PILOT-TESTING OF RAPID ASSESSMENT TOOL FOR PRE-HOSPITAL CARE IN KAMPALA, UGANDA}

${ }^{1}$ Amber Mehmood, ${ }^{1}$ Nino Paichadze, ${ }^{2}$ Esther Bayiga, ${ }^{1}$ Alexander Sloboda, ${ }^{3}$ Tonny Luggya, ${ }^{4}$ Joseph Kalanzi, ${ }^{2}$ Olive Kobusingye. ${ }^{1} J$ ohns Hopkins Bloomberg School of Public Health, USA; ${ }^{2}$ Makerere University School of Public Health, Uganda; ${ }^{3}$ Mulago National Referral Hospital, Uganda; ${ }^{4}$ Ministry of Health Republic of Uganda

\subsection{6/injuryprev-2016-042156.594}

Background Injury burden is disproportionately high in low- and middle-income countries (LMICs) but the healthcare systems are least prepared to meet the challenge. Significant gaps exist in emergency care, with most preventable deaths occurring in the pre-hospital phase due to lack of emergency medical services (EMS). There are no tools to define gaps and prioritise interventions in pre-hospital care. Realising this gap, our study was conducted to: i) develop a rapid assessment tool to define the structures, resources and processes comprising EMS; ii) pilot test the tool in Kampala, Uganda; and iii) identify gaps and provide recommendations for the improvement of EMS in Uganda.

Methods The study comprised of three phases: in Phase I rapid assessment tool was developed using the health systems framework with six building blocks; in Phase II a $360^{\circ}$ evaluation was performed by conducting relevant document review, objective assessment of ambulance services representing various levels of pre-hospital care and qualitative assessment through key informant interviews (KIIs) and focus group discussions (FGDs). Phase III, data analysis is underway.

Results The data was collected using the structured tool. Three study personnel reviewed 15 documents for the study. Three data collectors simultaneously performed 20 purposefully sampled KIIs and objective assessment. In addition, 4 FGDs will be completed by November 2015. The pilot study of the rapid assessment tool demonstrated the reliability, accuracy and completeness of the tool; further analysis after FDGs will allow us to describe the current state of EMS in Kampala. Based on the findings, context-specific interventions will be recommended.

Conclusions There is an immediate need to understand the contextual barriers of providing systematic emergency care in LMICs. The study has successfully developed an EMS assessment tool, which will also help establish strategies for improvement of pre-hospital care in similar settings.

\section{HELMET USE AND SEVERITY OF INJURY IN MOTORCYCLIST PATIENTS IN KHON KAEN HOSPITAL, THAILAND}

Porntipa Tantibundit, Arun Woranuch. Emergency Department, Khon Kaen Hospital, Thailand

\subsection{6/injuryprev-2016-042156.595}

Background Motorcycle is the most common vehicle use in Thailand because of topography, traffic jam problem and income of the owner. The percentage of motorcyclists lower than 20 years and over 60 years are increase every year. Among traffic accidents, increasing numbers of motorcycle accidents have been observed and it is the common cause of death in Thai population. This study aimed to identify severity of injury and helmet use in motorcycle patients in Khon Kaen hospital among teenage, adult and elderly groups.

Methods This was the retrospective cohort study. The data was extracted from the Electronic Injury Surveillance System that identified the patients who visited emergency department Khon Kaen hospital to treat injuries resulting from motorcycle accident in $1^{\text {st }}$ January $2013-31^{\text {th }}$ December 2104. Helmet use and severity of injuries sustained among those age lower than 20 years, 20-59 years, 60 years and older were compared.

Results 3,265 patients were lower than 20 years, 8,510 patients were 20-59 years and 737 patients were 60 years and older. All were treated at emergency department. The helmet used in lower than 20 years group and 60 years and older group were lower than $20-59$ years group (RR 0.73 ; 95\% CI: $0.68-0.79$ ) and (RR 0.76 ; 95\% CI: $0.66-0.88)$. Glasgow coma scale $\leq 7$ in lower than 20 years group and 60 years and older group were higher than 20-59 years group (RR 1.64; 95\% CI: 1.33-2.08) and (RR 4.16; 95\% CI: 3.44-5.00). Admission rate in 60 years and older group was higher when compared to 20-59 years group (RR 1.38; $95 \%$ CI: $1.29-1.47$ p-value $<0.00001)$ and also in hospital mortality in oldest group was about 1.7 fold when compared to 20-59 years group (RR 1.7; 95\% CI: $1.15-2.51 \mathrm{p}$ value $=0.00074$ ).

Conclusions The helmet used in younger and older patients were low and because of the older with motorcycle accident were prone to more severe injuries than younger adults. In future, the injury and prevention program should focus on this aged group. 